\title{
Approximations of Variational Problems in Terms of Variational Convergence
}

\author{
Huynh Thi Hong Diem
}

\begin{abstract}
We show first the definition of variational convergence of unifunctions and their basic variational properties. In the next section, we extend this variational convergence definition in case the functions which are defined on product two sets (bifunctions or bicomponent functions). We present the definition of variational convergence of bifunctions, icluding epi/hypo convergence, minsuplop convergnece and maxinf-lop convergence, defined on metric spaces. Its variational properties are also considered. In this paper, we concern on the properties of epi/hypo convergence to apply these results on optimization proplems in two last sections. Next we move on to the main results that are approximations of typical and important optimization related problems on metric space in terms of the types of variational convergence are equilibrium problems, and multiobjective optimization. When we applied to the finite dimensional case, some of our results improve known one.
\end{abstract}

Index Terms- Variational convergence variational properties saddle points equilibrium problems multiobjective optimization

\section{INTRODUCTION}

$\mathrm{V}$ ariational convergence has been considered for half a century with many important applications because it preserves variational properties. This preservation means that, when a sequence of functions converges to a limit function, properties such as being infimum or supremum values, minimizers, maximizes, minsup or maxinf values, minsup-points, saddle points, etc, of these

Manuscript Received on July 13 ${ }^{\text {th }}$, 2016. Manuscript Revised December $06^{\text {th }}, 2016$.

This research is funded by Ho Chi Minh City University of Technology - VNU-HCM under grant number T-KUD-2017-33

Huynh Thi Hong Diem, Department of Mathematics, Ho Chi Minh City University of Technology, Vietnam National University Hochiminh City, Vietnam.

Email: hthdiem@hcmut.edu.vn functions are inherited by the limit functions. These objects have played important roles in variational analysis and optimization-related problems. Hence, variational convergence is vital for studying approximation, estimation, stability or sensitivity in these areas. For unicomponent functions (unifunctions, or simply, functions), the basic variational convergence is epi-convergence, which was introduced in [1-3]. For bicomponent functions (or simply, bifunctions), i.e., functions of two components, and one wants to minimize with respect to (wrt) one component and maximize wrt the other. The first variational convergence is epi/hypo convergence proposed in [4,5] and the second notion is lopside convergence defined in [6]. In [7,8] lopside convergence of finite-valued bifunctions defined on rectangles (i.e., product sets) was introduced and studied with applications in approximation/stability of variational problems. In $[9,10]$ epi/hypo convergence of finite-valued bifunctions defined on rectangles was developed for the finite-dimensional case. For unifunctions the books [11,12] are prominent comprehensive references. Epi-convergence is used in $[11,12,13]$ for scalar minimization, graphical convergence is applied in [11] for complementarity problems and in [14] for variational inequalities, and lopconvergence is the tool in $[15,9,10,16]$ for various models. Epi/hypo convergence is studied and applied in $[17,18,10,19]$.

Regarding the notation, for a subset $A$ of a metric space $X$, int $A$ and $\operatorname{bd} A$ stand for its interior and boundary, respectively. For a function $\psi: X \rightarrow \overline{\mathrm{R}}:=\mathrm{R} \cup\{+\infty\}, \quad \liminf \psi$ and $\limsup \psi$ designate the lower and upper limits of $\psi$ as $x$ tends to $\bar{x}$. A function $\psi: \mathrm{R}^{n} \rightarrow \overline{\mathrm{R}}$ is said to be lower (upper, resp) semicontinuous (abbreviated as lsc (usc, resp)), at $\bar{x}$ if $\liminf _{x \rightarrow \bar{x}} \psi(x) \geq \psi(\bar{x})$ (limsup $x \rightarrow \bar{x} \psi(x) \leq \psi(\bar{x}))$. For a sequence of subsets $A^{k}$ in $X$, the lower/inner limit and upper/outer limit are defined by

$$
\operatorname{Liminf}_{k} A^{k}:=\left\{x \in X \mid \exists x^{k} \rightarrow x \text { with } x^{k} \in A^{k}\right\},
$$




$$
\begin{aligned}
& \operatorname{Limsup}_{k} A^{k}:=\left\{x \in X \mid \exists k_{l}\right. \text { (a subsequence), } \\
& \left.\exists x^{k} \rightarrow x \text { with } x^{k}{ }^{k} \in A^{k}\right\} .
\end{aligned}
$$

If $\operatorname{Liminf}_{k} A^{k}=\operatorname{Limsup}_{k} A^{k}$, one says that $A^{k}$ tends to $A$ or $A=\operatorname{Lim}_{k} A^{k}$ (in the PainlevéKuratowski sense). In the sequel denote the collection of the finite-valued unifunctions by fvfen $(X)$, and we sometimes write simply li, ls, Li, Ls for liminf, limsup, Liminf, Limsup, resp.

\section{VARIATIONAL CONVERGENCE OF UNIFUNCTIONS}

In this section, we discuss types of variational convergence of functions in $\mathrm{fv}-\mathrm{fcn}(X)$ and their basic variational properties. From now on in this paper, let $A^{k}, A \subset X$ be nonempty and $\left\{\varphi^{k}: A^{k} \rightarrow \mathrm{R}\right\}_{k}, \varphi: A \rightarrow \mathrm{R}$.

Definition 1 (Epi-convergence, [7]) $\left\{\varphi^{k}\right\}_{k}$, is called epi-convergent to $\varphi$, denoted by $\varphi=\mathrm{e}-$ $\lim _{k} \varphi^{k}$ or $\varphi^{k} \stackrel{e}{\rightarrow} \varphi$ if the following conditions are satisfied

(a) for all $x^{k} \in A^{k} \rightarrow x, \liminf _{k} \varphi^{k}\left(x^{k}\right) \geq \varphi(x)$ when $x \in A$ and $\varphi^{k}\left(x^{k}\right) \rightarrow+\infty$ when $x \notin A$;

(b) for all $x \in A$, there exists $x^{k} \in A^{k} \rightarrow x$ such that $\limsup _{k} \varphi^{k}\left(x^{k}\right) \leq \varphi(x)$.

$\left\{\varphi^{k}\right\}_{k}$ is called hypo-convergent to $\varphi$, denoted by $\varphi=\mathrm{h}-\lim _{k} \varphi^{k}$ or $\varphi^{k} \stackrel{h}{\rightarrow} \varphi$ if $-\varphi^{k}$ epi-converge to $-\varphi$.

Proposition 1 Let $A^{k}, A \subset X$ be nonempty and $\left\{\varphi^{k}: A^{k} \rightarrow \mathrm{R}\right\}_{k}, \varphi: A \rightarrow \mathrm{R}$.

(i) ([12], Proposition 5.33) $\operatorname{Lim}_{k} \operatorname{ghp} \varphi^{k}=\mathrm{g} p h \varphi$ (i.e., $\varphi^{k}$ graphically converge to $\varphi$ ) if and only if the following two conditions hold, for all $x \in A$,

( $\alpha$ ) for all $x^{k} \in A^{k} \rightarrow x$ there exists a subsequence $x^{k} j$ such that $\lim _{j} \varphi^{k_{j}}\left(x^{k}\right)=\varphi(x)$;

( $\beta$ ) there exists $x^{k} \in A^{k} \rightarrow x$ such that $\lim _{k} \varphi^{k}\left(x^{k}\right)=\varphi(x)$.

(ii) $\varphi^{k}$ graphically converge to $\varphi$ if and only if they both epi- and hypo-converge to $\varphi$ and $\lim _{k} A^{k}=A$.

(iii) If $\left\{\varphi^{k}\right\}_{k}$ converges continuously to $\varphi$ relative to sequence $A^{k} \rightarrow A$, then it both epi- and hypo-converges to $\varphi$. Proof (ii) Use the characterization by conditions $(\alpha)$ and $(\beta)$ in (i) for graphical convergence. (iii) This is clear. W

In the rest of this section, we recall most important variational properties of epiconvergence, see [7].

Theorem 1 (Epi-convergence: basic property) Let $\varphi^{k}, \varphi \in f v-f c n(X)$ and $\varphi=\mathrm{e}-\lim \varphi^{k}$. Then,

$$
\limsup _{k}\left(\inf _{A}{ }^{k} \varphi^{k}(x)\right) \leq \inf _{A} \varphi(x) \text {. }
$$

Moreover, if $x^{k}$ is a minimizer of $\varphi^{k}$ for some subsequence $\left\{k_{j}\right\}_{j}$ and $x^{k} \rightarrow \bar{x} \in A$, then $\bar{x}$ is a minimizer of $\varphi$ and the minimal values tend to that of $\varphi$.

The second part of Theorem 1 can be expressed equivalently as: if $\operatorname{e}^{-\lim _{k}} \varphi^{k}=\varphi$ then

$$
A \cap \operatorname{Limsup}_{k}\left(\operatorname{argmin}_{A} \varphi^{k}\right) \subset \operatorname{argmin}_{A} \varphi .
$$

It is easy to prove the extension that if $\left.\varepsilon^{k}\right] 0$ then

$$
A \cap \operatorname{Limsup}_{k}\left(\varepsilon^{k}-\operatorname{argmin}_{A^{k}} \varphi^{k}\right) \subset \operatorname{argmin}_{A} \varphi .
$$

To guarantee the equality in this relation with the full Lim instead of Limsup and also the convergence of the infimal values, we need the following tightness notion.

Definition 2 (Tight epi-convergence) We say that a sequence $\left\{\varphi^{k}\right\}_{k}$ epi-converges tightly to $\varphi$ in $\mathrm{fv}-\mathrm{fcn}(X)$ if it epi-converges and, for all positive $\varepsilon$, there exists a compact set $B_{\varepsilon}$ and an index $k_{\varepsilon}$ such that, for all $k \geq k_{\varepsilon}$,

$$
\inf _{B_{\varepsilon} \cap A^{k}} \varphi^{k} \leq \inf { }_{A}^{k} \varphi^{k}+\varepsilon .
$$

Theorem 2 (Convergence of infima) Let $\varphi^{k}, \varphi \in$

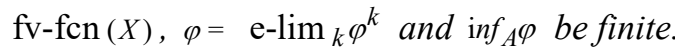

Then, the epi-convergence is tight

(i) if and only if $\inf _{A^{k}} \varphi^{k} \rightarrow \inf _{A} \varphi$;

(ii) if and only if there exists a sequence $\left.\varepsilon^{k}\right] 0$ such that $A \cap \operatorname{Lim}_{k} \varepsilon^{k}-\operatorname{argmin} \varphi^{k}=\operatorname{argmin} \varphi$.

\section{EPI/HYPO CONVERGENCE OF BICOMPONENT} FUNCTIONS AND VARIATIONAL PROPERTIES

We investigate $A, A^{k} \subset X, \quad B, B^{k} \subset Y$, $\Phi^{k}: A^{k} \times B^{k} \rightarrow \overline{\mathrm{R}}$, and $\Phi: A \times B \rightarrow \overline{\mathrm{R}}$

Definition 3 (Epi/hypo-convergence) 
Bifunctions $\Phi^{k}, k \in \mathrm{N}$, in fv-biv $(X \times Y)$ are called epi/hypo convergent (e/h-convergent) to a bifunction $\Phi \in$ fv-biv $(X \times Y)$ if

(a) for all $y \in B$ and all $x^{k} \in A^{k} \rightarrow x$, there exists $y^{k} \in B^{k} \rightarrow y$ such that $\liminf _{k} \Phi^{k}\left(x^{k}, y^{k}\right) \geq \Phi(x, y)$ if $x \in A$ or $\Phi^{k}\left(x^{k}, y^{k}\right) \rightarrow+\infty$ if $x \notin A$;

(b) for all $x \in A$ and all $y^{k} \in B^{k} \rightarrow y$, there exists $x^{k} \in A^{k} \rightarrow x$ such that $\limsup _{k} \Phi^{k}\left(x^{k}, y^{k}\right) \leq \Phi(x, y)$ if $y \in B$ or $\Phi^{k}\left(x^{k}, y^{k}\right) \rightarrow-\infty$ if $y \notin B$.

We denote this convergence by $\Phi=\mathrm{e} / \mathrm{h}-\lim _{k} \Phi^{k}$ or $\Phi^{k} \stackrel{e / h}{\rightarrow} \Phi$. Note that if the functions $\Phi^{k}$ do not depend on $y$, then epi/hypo convergence reduces to epi-convergence, and if they do not depend on $x$, it collapses to hypo-convergence. However, note that epi/hypo convergence is not epiconvergence of the $\Phi^{k}(\cdot, y)$ to $\Phi(\cdot, y)$ for all $y$ and hypo-convergence of the $\Phi^{k}(x, \cdot)$ to $\Phi(x$,$) for all x$. This is a sufficient condition for e/h-convergence, but not necessary. Indeed, $\Phi^{k}$ in Example 1 below e/h-converge, but it does not hold that $\Phi^{k}(\cdot, y) \stackrel{e}{\rightarrow} \Phi(\cdot, y)$ for all $y \in B$. It should be noticed also that the definitions of epi/hypo and hypo/epi convergence are symmetric. Furthermore, hypo/epi convergence coincides with epi/hypo convergence, if we insist minimizing in $x$ and maximizing in $y$, changing only the order of these two operations. (This fact was not noted clearly in existing papers in the literature.) To see that this symmetry is an important feature of epi/hypo convergence, let us recall the following.

Definition 4 (Minsup-lop convergence, [7]) Bifunctions $\Phi^{k} \in \mathrm{fv}$-biv $(X \times Y)$ are said to minsuplopside converge (minsup-lop converge) to $\Phi \in \mathrm{fv}$ $\operatorname{biv}(X \times Y)$ if

(a) for all $y \in B$ and $x^{k} \in A^{k} \rightarrow x$, there exists $y^{k} \in B^{k} \rightarrow y$ such that $\liminf _{k} \Phi^{k}\left(x^{k}, y^{k}\right) \geq \Phi(x, y)$ if $x \in A$ or $\Phi^{k}\left(x^{k}, y^{k}\right) \rightarrow \infty$ if $x \notin A$;

(b) for all $x \in A$, there exists $x^{k} \in A^{k} \rightarrow x$ such that, for all $y^{k} \in B^{k} \rightarrow y, \limsup _{k} \Phi^{k}\left(x^{k}, y^{k}\right) \leq \Phi(x, y)$ if $y \in B$ or $\Phi^{k}\left(x^{k}, y^{k}\right) \rightarrow-\infty$ if $y \notin B$.

Observe that Definition 4 is nonsymmetric: the following maxinf-lop convergence is different from minsup-lop convergence: (a) for all $x \in A$ and $y^{k} \in B^{k} \rightarrow y$, there exists $x^{k} \in A^{k} \rightarrow x$ such that $\limsup _{k} \Phi^{k}\left(x^{k}, y^{k}\right) \leq \Phi(x, y)$ if $y \in B$ or $\Phi^{k}\left(x^{k}, y^{k}\right) \rightarrow-\infty$ if $y \notin B$;

(b) for all $y \in B$, there exists $y^{k} \in B^{k} \rightarrow y$ such that, for all $x^{k} \in A^{k} \rightarrow x, \liminf _{k} \Phi^{k}\left(x^{k}, y^{k}\right) \geq \Phi(x, y)$ if $x \in A$ or $\Phi^{k}\left(x^{k}, y^{k}\right) \rightarrow \infty$ if $x \notin A$.

Lopside convergence clearly implies e/hconvergence. Indeed, condition (a) of the definitions are the same whereas condition (b) of lop-convergence is clearly stronger than (b) of epi/hypo convergence. To see this, simply observe that, if for all $x \in C$ one can find a common sequence $\quad\left\{x^{k} \in A^{k}\right\}_{k \in \mathrm{N}} \quad$ such that $\limsup _{k} \Phi^{k}\left(x^{k}, y^{k}\right) \leq \Phi(x, y) \quad$ or $\quad \Phi^{k}\left(x^{k}, y^{k}\right) \rightarrow-\infty$ depending on $y$ belonging or not to $B$ as lopconvergence requires, then certainly (b) for epi/hypo convergence is satisfied, since one can even choose such a sequence $x^{k} \rightarrow x$ to depend on $y^{k} \rightarrow y$. However, the converse does not hold as shown by the following.

Example 1 Let $A^{k}=B^{k}=[1 / k, 1], A=B=[0,1]$, and

$$
\Phi^{k}(x, y)= \begin{cases}1 & \mathrm{i} f(x, y) \in A^{k} \times B^{k} \text { and } x \neq y, \\ 0 & \mathrm{i} f(x, y) \in A^{k} \times B^{k} \text { and } x=y .\end{cases}
$$

Then,

$$
\Phi(x, y)= \begin{cases}1 & \text { if }(x, y) \in[0,1] \text { and } x \neq y, \\ 0 & \text { if } f(x, y) \in[0,1] \text { and } x=y .\end{cases}
$$

Clearly $\Phi^{k} \stackrel{e / h}{\rightarrow} \Phi$. We show that condition (b) of Definition 4 of minsup-lop convergence is violated. For $x=0$ and any $x^{k} \in A^{k} \rightarrow x$, we take $y=0$ and $y^{k} \in B^{k} \rightarrow 0$ such that $y^{k} \neq x^{k}$ for all $k$. Then, $\limsup _{k} \Phi^{k}\left(x^{k}, y^{k}\right)=1>0=\Phi(x, y)$.

\section{Remark 1}

(i) It is clear that continuous convergence of bifunctions $\Phi^{k}(,):, A^{k} \times B^{k} \rightarrow \mathrm{R}$ relative to the sequence $A^{k} \times B^{k} \rightarrow A \times B$ implies all kinds of e/h-, minsup-lop and maxinf-lop convergence. (We know already in Section 2 that continuous convergence implies also both epi- and hypoconvergence of $\Phi^{k}(\cdot$,$) .) So, continuous$ convergence is a variational convergence too. But, this convergence is very strong and hence difficult to be satisfied.

(ii) Limits of an e/h-convergent sequence are not unique. The limits form a class of bifunctions, called an e/h-equivalence class ; see e.g., [9]. However, as we will see below, fortunately almost 
all variational properties are the same for all the limit bifunctions in an equivalence class.

(iii) In [9], characterizations of e/h-convergence and lop-convergence of finite-valued bifunctions were established. In particular, [9, Theorem 3] asserted the equivalence of the e/h-convergence of a sequence of finite-valued bifunctions and the e/hconvergence of the corresponding proper extendedreal-valued bifunctions.

Naturally expected variational properties of e/hconvergence are those related to saddle points, since this convergence is symmetric. Recall that a point $(\bar{x}, \bar{y}) \in A \times B$ is said to be a saddle point of $\Phi \in$ fv-biv $(X \times Y)$, denoted by $(\bar{x}, \bar{y}) \in \mathrm{s} d l \Phi$, if, for all $x \in A$ and $y \in B$, $\Phi(\bar{x}, y) \leq \Phi(\bar{x}, \bar{y}) \leq \Phi(x, \bar{y})$,

or equivalently, $\Phi(\bar{x}, y) \leq \Phi(x, \bar{y}) \quad$ for all $(x, y) \in A \times B$.

In applications, approximate saddle points often exist even when saddle points do not. Hence, we will prove the following convergence of approximate saddle points. The convergence of saddle points will follows immediately. Recall that, for a nonegative $\varepsilon$, a point $(\bar{x}, \bar{y}) \in A \times B$ is said to be an $\varepsilon$-saddle point of $\Phi \in \mathrm{fv}$-biv $(X \times Y)$, denoted by $(\bar{x}, \bar{y}) \in \varepsilon-\operatorname{sdl} \Phi$, if, for all $x \in A$ and $y \in B$,

$$
\Phi(\bar{x}, y)-\varepsilon \leq \Phi(\bar{x}, \bar{y}) \leq \Phi(x, \bar{y})+\varepsilon,
$$

or equivalently, $\Phi(\bar{x}, y)-\varepsilon \leq \Phi(x, \bar{y})+\varepsilon$ for all $(x, y) \in A \times B$.

Let us define the sup-projection and infprojection of a bifunction $\Phi \in \operatorname{fv}-\operatorname{biv}(X \times Y)$ by, resp,

$$
\varsigma(.):=\sup _{y \in B} \phi(., y), \quad \eta(.):=\mathrm{inf} f_{x \in A} \Phi(x, .) .
$$

We have the following simple relation between approximate solutions.

Proposition 2 Let $\Phi \in \mathrm{fv}$-biv $(X \times Y)$ and $\varsigma$ and $\eta$ be its sup-projection and inf-projection, respectively.

(i) If $(\bar{x}, \bar{y}) \in \varepsilon-\operatorname{sdl} \Phi$, then $\bar{x} \in 2 \varepsilon-\operatorname{argmin}(\varsigma)$ and $\bar{y} \in 2 \varepsilon-\operatorname{argmax}(\eta)$.

(ii) If $\bar{x} \in \varepsilon-\operatorname{argmin}(\varsigma)$ and $\bar{y} \in \varepsilon-\operatorname{argmax}(\eta)$, then

$$
\eta(\bar{y}) \leq \Phi(\bar{x}, \bar{y}) \leq \varsigma(\bar{x}),
$$

$\sup _{y \in B} \inf _{x \in A} \Phi(x, y)-\varepsilon \leq \Phi(\bar{x}, \bar{y}) \leq \inf _{x \in A} \sup _{y \in B} \Phi(x, y)+\varepsilon$.

Therefore, if $\Phi$ has a saddle point $(\tilde{x}, \tilde{y})$, then $\Phi(\tilde{x}, y)-\varepsilon \leq \Phi(\bar{x}, \bar{y}) \leq \Phi(x, \tilde{y})+\varepsilon$.

Proof (i) We have

$$
\varsigma(\bar{x})=\sup _{y \in B} \Phi(\bar{x}, y)=\Phi(\bar{x}, \bar{y})+\varepsilon
$$

$$
=\inf _{x \in A} \Phi(x, \bar{y})+2 \varepsilon \leq \inf _{x \in A} \sup _{y \in B} \Phi(x, y)+2 \varepsilon=\inf _{x \in A} \varsigma(x)+2 \varepsilon .
$$

The corresponding property of $\eta$ is checked similarly, (ii) It is clear that

$$
\Phi(\bar{x}, \bar{y}) \geq \eta(\bar{y}) \geq \sup _{y \in B} \inf _{x \in A} \Phi(x, y)-\varepsilon .
$$

The two right inequalities are proved similarly. Finally, if $(\widetilde{x}, \widetilde{y}) \in \mathrm{s} d l \Phi$, then

$$
\Phi(\tilde{x}, y)-\varepsilon \leq \Phi(\tilde{x}, \tilde{y})-\varepsilon \leq \Phi(\bar{x}, \bar{y}) \leq \Phi(\tilde{x}, \tilde{y})+\varepsilon \leq \Phi(x, \tilde{y})+\varepsilon .
$$

In the remaining part of this section, we investigate variational properties of an arbitrary e/h-limit under some additional conditions. We will see that all the e/h-limits in an equivalence class share many common properties. This fact should be highlighted, since in many applications it helps to avoid dealing with whole equivalence classes.

Theorem 3 (Convergence of approximate saddle points) Let a sequence $\left\{\Phi^{k}\right\}_{k}$ e/h-converge to $\Phi$ in fv-biv $\left.(X \times Y), \varepsilon^{k}\right] \varepsilon \geq 0$ and, for all $k \in \mathrm{N}$, $\left(\bar{x}^{k}, \bar{y}^{k}\right) \in \varepsilon^{k}-\operatorname{sdl} \Phi^{k}$. Let $(\bar{x}, \bar{y}) \in A \times B$ be a cluster point of this sequence of approximate saddle points, say $(\bar{x}, \bar{y})=\lim _{k}\left(\bar{x}^{k}, \bar{y}^{k}\right)$ for some subsequence $N \subset \mathrm{N}$. Then, $(\bar{x}, \bar{y})$ is an $\varepsilon$-saddle point of $\Phi$ and

$$
\Phi(\bar{x}, \bar{y})=\lim _{k} \Phi^{k}\left(\bar{x}^{k}, \bar{y}^{k}\right) .
$$

Proof We can assume that actually $\left(\bar{x}^{k}, \bar{y}^{k}\right) \rightarrow(\bar{x}, \bar{y})$. Pick any $(x, y) \in A \times B$. Any sequences $x^{k} \in A^{k} \rightarrow x$ and $y^{k} \in B^{k} \rightarrow y$ satisfy

$$
\Phi^{k}\left(\bar{x}^{k}, y^{k}\right)-\varepsilon^{k} \leq \Phi^{k}\left(\bar{x}^{k}, \bar{y}^{k}\right) \leq \Phi^{k}\left(x^{k}, \bar{y}^{k}\right)+\varepsilon^{k} .
$$

These inequalities imply that

$$
\begin{aligned}
& \sup _{\left\{y^{k} \in B^{k} \rightarrow y\right\}} \operatorname{iminf}_{k}\left(\Phi^{k}\left(\bar{x}^{k}, y^{k}\right)-\varepsilon^{k}\right) \\
& \leq \liminf _{k} \Phi^{k}\left(\bar{x}^{k}, \bar{y}^{k}\right) \leq \limsup _{k} \Phi^{k}\left(\bar{x}^{k}, \bar{y}^{k}\right) \\
& \leq \inf _{\left\{x^{k} \in A^{k} \rightarrow x\right\}} \limsup _{k}\left(\Phi^{k}\left(x^{k}, \bar{y}^{k}\right)+\varepsilon^{k}\right),
\end{aligned}
$$

By the definition of e/h-convergence, one has

$$
\begin{aligned}
& \Phi(\bar{x}, y)-\varepsilon \leq \sup _{\left\{y^{k} \in B^{k} \rightarrow y\right\}} \liminf _{k}\left(\Phi^{k}\left(\bar{x}^{k}, y^{k}\right)-\varepsilon^{k}\right) \\
\leq & \inf f_{\left\{x^{k} \in A^{k} \rightarrow x\right\}} \limsup _{k}\left(\Phi^{k}\left(x^{k}, \bar{y}^{k}\right)+\varepsilon^{k}\right) \leq \Phi(x, \bar{y})+\varepsilon .
\end{aligned}
$$

These inequalities mean that $(\bar{x}, \bar{y})$ is an $\varepsilon$ saddle point of $\Phi$. To see that $\Phi(\bar{x}, \bar{y})=\lim _{k} \Phi^{k}\left(\bar{x}^{k}, \bar{y}^{k}\right)$, simply observe that the e/hconvergence and $\bar{x}^{k} \rightarrow \bar{x}$ ensure the existence of a sequence $y^{k} \in B^{k} \rightarrow \bar{y}$ satisfying

$$
\begin{gathered}
\Phi(\bar{x}, \bar{y}) \leq \liminf _{k} \Phi^{k}\left(\bar{x}^{k}, y^{k}\right) \\
\leq \liminf _{k}\left(\Phi^{k}\left(\bar{x}^{k}, \bar{y}^{k}\right)+\varepsilon^{k}\right)=\liminf _{k} \Phi^{k}\left(\bar{x}^{k}, \bar{y}^{k}\right),
\end{gathered}
$$


where the second inequality follows from the approximate saddle point inequalities. With the role played by the $x$-variable and the $y$-variable reversed, a similar argument gives

$$
\Phi(\bar{x}, \bar{y}) \geq \underset{k \rightarrow \infty}{\limsup } \Phi^{k}\left(\bar{x}^{k}, \bar{y}^{k}\right) .
$$

Clearly, by taking $\varepsilon^{k} \equiv 0$ in the preceding statement, we obtain the following basic result on convergence of saddle points.

Theorem 4 (Convergence of saddle points) Let a sequence $\left\{\Phi^{k}\right\}_{k}$ e/h-converge to $\Phi$ in $\mathrm{fv}$ $\operatorname{biv}(X \times Y)$ and $\left(\bar{x}^{k}, \bar{y}^{k}\right)$ be a saddle point of $\Phi^{k}$ for all $k \in \mathrm{N}$. Let $(\bar{x}, \bar{y}) \in A \times B$ be a cluster point of this sequence of saddle points, say $(\bar{x}, \bar{y})=\lim _{k}\left(\bar{x}^{k}, \bar{y}^{k}\right)$ for some subsequence $N \subset \mathrm{N}$. Then, $(\bar{x}, \bar{y})$ is a saddle point of $\Phi$ and

$$
\Phi(\bar{x}, \bar{y})=\lim _{k} \Phi^{k}\left(\bar{x}^{k}, \bar{y}^{k}\right) .
$$

Observe that, in the above two theorems, neither convex-concave conditions, nor continuity, nor compactness, nor even closedness are imposed. We assume only epi/hypo convergence. So, this convergence is a very suitable notion for considering saddle (or approximate saddle) points.

Theorem 3 can be restated as follows, for the case $\varepsilon=0$. If $\Phi^{k} \mathrm{e} / \mathrm{h}$-converges to $\Phi$ and $\left.\varepsilon^{k}\right] 0$, then

$$
(A \times B) \cap \operatorname{Limsup}_{k}\left(\varepsilon^{k}-\operatorname{sdl} \Phi^{k}\right) \subset \operatorname{s} d l \Phi .
$$

To have equality with the full Lim instead of Limsup in the above relation, i.e., to have also

$$
(A \times B) \cap \operatorname{Liminf}_{k}\left(\varepsilon^{k}-\mathrm{sdl} \Phi^{k}\right) \supset \mathrm{s} d l \Phi,
$$

we propose new notions of tightness in Definition 5 below. Note that these tightness definitions reflect the symmetric roles of $x$ and $y$ in the symmetric e/h-convergence (cf. discussions after Definition 3). They are different from the known notions of tightness in [7] which are nonsymmetric.

\section{Definition 5}

(i) ( $x$-ancillary tightness) $\Phi^{k}$ is called to e/hconverge $x$-ancillary tightly to $\Phi$ in fv-biv $(X \times Y)$ if (a) of Definition 3 and the following condition are satisfied:

(b'-t) for all $x \in A$, there is $x^{k} \in A^{k} \rightarrow x$ such that $\mathrm{h}-\lim _{k} \Phi^{k}\left(x^{k},\right)=\Phi(x, ;)$ and $\varsigma^{k}\left(x^{k}\right) \rightarrow \varsigma(x)$.

(ii) ( $y$-ancillary tightness) $\Phi^{k}$ is said to e/hconverge $y$-ancillary tightly to $\Phi$ in fv-biv $(X \times Y)$ if (b) of Definition 3 is fulfilled together with (a'-t) for all $y \in B$, there is $y^{k} \in B^{k} \rightarrow y$ such that $\mathrm{e}-\lim _{k} \Phi^{k}\left(\cdot, y^{k}\right)=\Phi(\cdot, y)$ and $\eta^{k}\left(y^{k}\right) \rightarrow \eta(y)$.

(iii) (tightness) If both (b'-t) and (a'-t) are satisfied, $\Phi^{k}$ is called to e/h-converge (fully) tightly to $\Phi$.

Theorem 5 (Convergence of approximate saddle points to any given saddle point) Suppose that $\Phi^{k}$ e/h-converges (fully) tightly to $\Phi$ in $\mathrm{fv}$ $\operatorname{biv}(X \times Y)$. Then, the following statements hold.

(i) $\operatorname{sdl} \Phi \subset \cap_{\varepsilon>0} \operatorname{Liminf}_{k}\left(\varepsilon-\operatorname{sdl} \Phi^{k}\right)$.

(ii) Therefore, for each $\left.\varepsilon^{k}\right] 0,(\bar{x}, \bar{y}) \in \mathrm{s} d l \Phi$ and large $k$, there exists $\left(\bar{x}^{k}, \bar{y}^{k}\right) \in \varepsilon^{k}-\operatorname{sdl} \Phi^{k}$ such that $\left(\bar{x}^{k}, \bar{y}^{k}\right) \rightarrow(\bar{x}, \bar{y})$, i.e.,

$$
(A \times B) \cap \operatorname{Liminf}_{k}\left(\varepsilon^{k}-\operatorname{sdl} \Phi^{k}\right) \supset \mathrm{s} d l \Phi .
$$

Proof (i) For each $(\bar{x}, \bar{y}) \in \mathrm{s} d l \Phi$, the tightness ensures the existence of $\bar{x}^{k} \in A^{k} \rightarrow \bar{x} \quad$ and $\bar{y}^{k} \in B^{k} \rightarrow \bar{y}$ such that

$$
\begin{aligned}
& \mathrm{h}-\lim _{k} \Phi^{k}\left(x^{k},\right)=\Phi(x, \cdot), \varsigma^{k}\left(\bar{x}^{k}\right) \rightarrow \varsigma(\bar{x}), \\
& e-\lim _{k} \phi^{k}\left(\cdot, y^{k}\right)=\phi(\cdot, y), \eta^{k}\left(\bar{y}^{k}\right) \rightarrow \eta(\bar{y}) .
\end{aligned}
$$

It suffices to show that, for all positive $\varepsilon$ and large $k$,

$$
\begin{aligned}
& \Phi^{k}\left(\bar{x}^{k}, \bar{y}^{k}\right) \geq \sup _{y \in B^{k}} \Phi^{k}\left(\bar{x}^{k}, y\right)-\varepsilon, \\
& \Phi^{k}\left(\bar{x}^{k}, \bar{y}^{k}\right) \leq \inf _{x \in A^{k}} \Phi^{k}\left(x, \bar{y}^{k}\right)+\varepsilon .
\end{aligned}
$$

Suppose to the contrary to (3) that there is $\varepsilon_{0}$ and a subsequence $k_{j}$ such that

$$
\Phi^{k_{j}}\left(\bar{x}^{k}{ }^{k}, \bar{y}^{k}{ }^{j}\right)<\sup _{y \in B} \Phi_{j} \Phi^{k_{j}}\left(\bar{x}^{k}{ }^{k}, y\right)-\varepsilon_{0} .
$$

Taking liminf on both sides, (1) and (2) imply that

$$
\Phi(\bar{x}, \bar{y}) \leq \liminf _{j} \Phi^{k}{ }_{j}\left(\bar{x}^{k}, \bar{y}^{k} j\right) \leq \sup _{y \in B} \Phi(\bar{x}, y)-\varepsilon_{0},
$$

which is impossible since $(\bar{x}, \bar{y}) \in \mathrm{s} d l \Phi$. Inequality (4) is similarly proved. (ii) Given $\left.\varepsilon_{\mu}\right] 0$ and a fixed $\mu$, by (i) one has a sequence $\left(\bar{x}_{\mu}^{k}, \bar{y}_{\mu}^{k}\right) \in \varepsilon_{\mu}-\operatorname{sdl} \Phi^{k}$ converging to $(\bar{x}, \bar{y})$. Using the diagonal technique we obtain the required sequence $\left(\bar{x}_{k}^{k}, \bar{y}_{k}^{k}\right)$ and complete the proof.

Example 2 Let $\Phi^{k}(x, y)=y^{x}$ on $[0,1]^{2}$ for all $k \in \mathrm{N}$, with the convention that $0^{0}=1$. In Example 2 of [9], it was computed that all the bifunctions 


$$
\Phi_{a}(x, y)=\left\{\begin{array}{cc}
y^{x} & \operatorname{if}(\mathrm{x}, \mathrm{y}) \in[0,1]^{2} \backslash\{(0,0)\}, \\
a & \operatorname{if}(\mathrm{x}, \mathrm{y})=(0,0),
\end{array}\right.
$$

for any $a \in[0,1]$, are $\mathrm{e} / \mathrm{h}$-limits of the sequence $\left\{\Phi^{k}\right\}$, i.e., they form the e/h-equivalence class. It is easy to check that $\mathrm{e} / \mathrm{h}-\lim _{k} \Phi^{k}=\Phi_{a}$ fully tightly. Evidently, each point in $\{(x, 1) \mid 0 \leq x \leq 1\}$ is a saddle point of all $\Phi^{k}$ and $\Phi_{a}$ for all $k \in \mathrm{N}$ and $a \in[0,1]$. So, the saddle points are preserved under tight e/hconvergence and the saddle points are the same for all limits in the e/h-equivalence class.

\section{APPROXIMATIONS OF EQUILIBRIUM PROBLEMS}

Consider the following equilibrium problem

(EP) find $\bar{x} \in A$ such that $\Phi(\bar{x}, y) \leq 0$ for all $y \in B$,

where $A \times B \subset X \times Y$ and $\Phi: A \times B \rightarrow \mathrm{R}$. The (EP) was shown to be a genuine generalization of many optimization-related problems in [20], by pointing out particular (EP) model which do not fit the framework of variational inequalities, which are also general but are special cases of (EP). Note that, in fact, we have not seen papers arguing if a solution of (EP) may be really a physical/technical equilibrium point or not. But, the economical and social meaning of "equilibrium" has been confirmed by many considerations of practical problems like traffic networks, non-cooperative games, etc.

Assume that for (EP) we have a sequence of approximating problems $\left(\mathrm{EP}^{k}\right)$ with $\Phi^{k}: A^{k} \times B^{k} \rightarrow \mathrm{R}$. Denote the solution set of (EP) $\left(\left(\mathrm{EP}^{k}\right)\right.$, resp) by $\mathrm{S}\left(\mathrm{S}^{k}\right.$, resp).

Proposition 3 If $\mathrm{e} / \mathrm{h}-\lim _{k} \Phi^{k}=\Phi$ or e$\lim _{k} \Phi^{k}=\Phi$, then

$$
(A \times B) \cap \text { Limsup }_{k} \mathrm{~S}^{k} \subset \mathrm{S},
$$

i.e., any cluster point in $A \times B$ of a sequence of solutions of problems $\left(\mathrm{EP}^{k}\right)$ is a solution of (EP).

Proof Let $\bar{x} \in A \cap \operatorname{Limsup}_{k} \mathrm{~S}^{k}$, i.e., there exists a sequence $\left\{x^{k}\right\}$ in $\mathrm{S}^{k}$ converging to $\bar{x}$. For any (fixed) $y \in B$, by (a) of Definition 3, there is a sequence $\left\{y^{k}\right\}_{j}$ in $B^{k}$ converging to $y$ such that $\quad \liminf _{j} \Phi^{k}{ }^{k}\left(x^{k} j, y^{k}\right) \geq \Phi(\bar{x}, y)$. Since $\Phi^{k} j(\bar{x}, y) \leq 0$ for all $j$ and $y \in B{ }^{k} j, \Phi^{k}{ }^{k}\left(x^{k} j, y^{k} j\right) \leq 0$ for all $j$ and hence $\liminf _{k_{j}} \Phi^{k}{ }^{k}\left(x^{k} j, y^{k}\right) \leq 0$. Thus, $\Phi(\bar{x}, y) \leq 0$ for any $y \in B$, i.e., $\bar{x}$ is in $\mathrm{S}$. The proof for the case where $\Phi^{k}$ epi-converges is similar. W

The above assertion improves Theorem 6.11 of [21], where the e/h-convergence is replaced by the stronger minsup-lop convergence, and it is assumed further that $A=B, A^{k}=B^{k}$ are closed, $A^{k} \times B^{k} \rightarrow A \times B$ and $\Phi^{k}, \Phi$ are lsc-usc for the case of minsup-lop convergence and lsc for the epi-convergence case.

The above statement can be extended to the case of approximate solutions as follows. Let $\xi: X \rightarrow \mathrm{R}$ be continuous such that $\xi(x)>0$ if $x \neq 0$. Consider (EP) with $m=n$ and $A=B$. Following Definition 7.1 in [21], for $\varepsilon \geq 0$, a point $\bar{x} \in A$ is called an $(\varepsilon, \xi)$-approximate solution of (EP) if $\Phi(\bar{x}, y) \leq \varepsilon \xi(\bar{x}-y)$ for all $y \in A$. Denote the set of all $(\varepsilon, \xi)$-approximate solutions of (EP) by $\mathrm{S}_{\varepsilon, \xi}$.

Proposition 4 If $\left.\varepsilon^{k}\right] \varepsilon \geq 0$ and $\mathrm{e} / \mathrm{h}-\lim _{k} \Phi^{k}=\Phi$ or $\mathrm{e}-\lim _{k} \Phi^{k}=\Phi$, then

$$
(A \times B) \cap \operatorname{Limsup}_{k} \mathrm{~S}_{\varepsilon^{k}, \xi}^{k} \subset \mathrm{S}_{\varepsilon, \xi},
$$

i.e., any cluster point in $A \times B$ of a sequence of $\left(\varepsilon^{k}, \xi\right)$-solutions of problems $\left(\mathrm{EP}^{k}\right)$ is an $(\varepsilon, \xi)$ solution of problem (EP).

The proof is similar to that of Proposition 3.

We consider also the dual equilibrium problem, introduced in [22],

(DEP) find $\bar{y} \in B$ such that $\Phi(x, \bar{y}) \geq 0$ for all $x \in A$.

Observe that (EP) and (DEP) are dual to each other, i.e., the dual of (DEP) is just (EP). To see clearer the essence of duality of these problems, we reformulate them as

(EP) find $\bar{x} \in A$ solving $\min _{x \in A} \sup _{y \in B} \Phi(x, y) \leq 0$,

(DEP) find $\quad \bar{y} \in B \quad$ solving $\max _{y \in B} \inf _{x \in A} \Phi(x, y) \geq 0$.

We have the following evident assertion, which is stronger than corresponding statements in many other duality schemes: $\bar{x}$ is a solution of (EP) and also $\bar{y}$ is that of (DEP) if and only if $(\bar{x}, \bar{y})$ is a saddle point of $\Phi$ in $A \times B$ and $\Phi(\bar{x}, \bar{y})=0$. Hence, we have zero duality gap for any couple of solutions $\bar{x}, \bar{y}$ of (EP) and (DEP), resp. Similarly, $\bar{x}_{\varepsilon, \xi}$ is an $(\varepsilon, \xi)$-approximate solution of (EP) and $\bar{y}_{\varepsilon, \xi}$ is that of (DEP) if and only if $\left(\bar{x}_{\varepsilon, \xi}, \bar{y}_{\varepsilon, \xi}\right)$ is an $(\varepsilon, \xi)$-approximate saddle point of $\Phi$ in $A \times B$ and $-\varepsilon \xi(\bar{x}-\bar{y}) \leq \Phi(\bar{x}, \bar{y}) \leq \varepsilon \xi(\bar{x}-\bar{y})$. Here, we naturally call $\left(\bar{x}_{\varepsilon, \xi}, \bar{y}_{\varepsilon, \xi}\right)$ an $(\varepsilon, \xi)$-approximate saddle point of $\Phi$ if 


$$
\Phi\left(\bar{x}_{\varepsilon, \xi}, y\right)-\varepsilon \xi\left(\bar{x}_{\varepsilon, \xi}-y\right) \leq \Phi\left(x, \bar{y}_{\varepsilon, \xi}\right)+\varepsilon \xi\left(x-\bar{y}_{\varepsilon, \xi}\right)
$$

for all $(x, y) \in A \times B$.

Suppose that (EP) and (DEP) have the approximating problems, called $\left(\mathrm{EP}^{k}\right)$ and $\left(\mathrm{DEP}^{k}\right)$, are stated in terms of $\Phi^{k}$ and $A^{k}, B^{k}$ instead of $\Phi$ and $A, B$. The following approximation result is an immediate consequence of Theorems 3 and 4.

Proposition 5 Let e/h-lim ${ }_{k} \Phi^{k}=\Phi$.

(i) If $\left.\varepsilon^{k}\right] \varepsilon \geq 0$ and $\bar{x}_{\varepsilon^{k}, \xi}^{k}$ and $\bar{y}_{\varepsilon^{k}, \xi}^{k}$ are an $\left(\varepsilon^{k}, \xi\right)-$ approximate solution of $\left(\mathrm{EP}^{k}\right)$ and $\left(\mathrm{DEP}^{k}\right)$, resp, then the two components of any cluster point $\left(\bar{x}_{\varepsilon, \xi}, \bar{y}_{\varepsilon, \xi}\right)$ in $A \times B$ of the sequence $\left\{\left(\bar{x}_{\varepsilon^{k}, \xi}^{k}, \bar{y}_{\varepsilon^{k}, \xi}^{k}\right)\right\}$ are an $(\varepsilon, \xi)$-approximate solution of (EP) and (DEP), resp, and one has

$$
-\varepsilon \leq \Phi\left(\bar{x}_{\varepsilon, \xi}, \bar{y}_{\varepsilon, \xi}\right)=\lim _{k} \Phi^{k}\left(\bar{x}_{\varepsilon^{k}, \xi}^{k}, \bar{y}^{k}{ }_{\varepsilon^{k}, \xi}^{k}\right) \leq \varepsilon .
$$

(ii) In particular, for $\varepsilon=0$ (i) becomes: if $\bar{x}_{\varepsilon^{k}, \xi}^{k}$ and $\bar{y}_{\varepsilon^{k}, \xi}^{k}$ are an $\left(\varepsilon^{k}, \xi\right)$-approximate solution of $\left(\mathrm{EP}^{k}\right)$ and $\left(\mathrm{DEP}^{k}\right)$, resp, then the two components of any cluster point $(\bar{x}, \bar{y})$ in $A \times B$ of the sequence

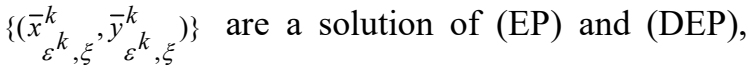
resp, and one has

$$
\Phi(\bar{x}, \bar{y})=\lim _{k} \Phi^{k}\left(\bar{x}_{\varepsilon^{k}, \xi^{k}}^{k}, \bar{y}_{\varepsilon^{k}, \xi}^{k}\right) .
$$

Denote the set of the solutions (the $(\varepsilon, \xi)$ approximate solutions, resp) of (DEP) by DS (DS ${ }_{\varepsilon, \xi}$, resp). Proposition 5 (ii) can be rephrased as follows: if $\left.\varepsilon^{k}\right] 0$ then

$$
(A \times B) \cap \operatorname{Limsup}_{k}\left(\mathrm{~S}_{\varepsilon^{k}, \xi}^{k} \times \mathrm{DS}_{\varepsilon^{k}, \xi}^{k}\right) \subset \mathrm{S} \times \mathrm{D} S .
$$

To have equality and with the "full" Lim instead of Limsup, i.e., to have additionally

$$
(A \times B) \cap \operatorname{Liminf}_{k}\left(\mathrm{~S}_{\varepsilon^{k}, \xi}^{k} \times \mathrm{DS}_{\varepsilon^{k}, \xi}^{k}\right) \supset \mathrm{S} \times \mathrm{D} S,
$$

we impose tightness conditions and apply Theorem 5 to obtain the following.

Proposition 6 If $\Phi^{k}$ e/h-converges fully tightly to $\Phi$ and $\left.\varepsilon^{k}\right] 0$, then

$$
(A \times B) \cap \operatorname{Lim}_{k}\left(\mathrm{~S}_{\varepsilon^{k}, \xi}^{k} \times \mathrm{DS}_{\varepsilon^{k}, \xi}^{k}\right)=\mathrm{S} \times \mathrm{D} S .
$$

\section{APPROXIMATIONS OF MULTIOBJECTIVE OPTIMIZATION}

As aforementioned, it is well-known that equilibrium models encompass most of optimization-related problems. However, in this paper we restrict ourselves to the particular equilibrium problem (EP), which is a single-valued and scalar problem. So, in principle, it contains only single-valued and scalar optimization-related models. But, we can apply Propositions 3-6 for our scalar problem (EP) to the following multiobjective minimization problem. Let $\varphi_{1}, \ldots, \varphi_{m}: A \subset X \rightarrow \mathrm{R}$ and $\mathrm{R}^{m}$ be ordered partially by $\mathrm{R}_{+}^{m}$. Our multiobjective minimization problem is

$$
\text { (OP) find } \bar{x} \in A \text { s.t. } \varphi(\bar{x})-\varphi(y) \notin \mathrm{int} \mathrm{R}_{+}^{m} \text { for all } y \in A \text {. }
$$

Such a $\bar{x}$ is called a weak minimizer (or weakly efficient point) of $\varphi$ on $A$. We can convert (OP) to a special case of (EP) by setting, see, e.g., [20],

$$
\Omega(x, y)=\min _{1 \leq i \leq m}\left(\varphi_{i}(x)-\varphi_{i}(y)\right) .
$$

Indeed, taking $B=A$, we have the three equivalent assertions, for all $y \in A$,

$$
\begin{aligned}
\Omega(\bar{x}, y) \leq 0 & \Leftrightarrow \text { thereexists } i, \varphi_{i}(\bar{x})-\varphi_{i}(y) \leq 0 \\
& \Leftrightarrow \varphi(\bar{x})-\varphi(y) \notin \mathrm{in} t \mathrm{R}_{+}^{m} .
\end{aligned}
$$

The dual to (OP) according to the duality scheme for (EP), i.e., problem (DEP) for $\Omega(x, y)=\min _{1 \leq i \leq m}\left(\varphi_{i}(x)-\varphi_{i}(y)\right)$, is

(DOP) find $\bar{y}$ such that $\varphi_{i}(x)-\varphi_{i}(\bar{y}) \geq 0$ for all $x \in A, i=1, \ldots, m$,

Such a solution $\bar{y}$ is called a strong/ideal minimizer (or a strongly efficient point) of $\varphi$ on $A$. This duality scheme is different from the known ones for multiobjective minimization, where the dual for a minimization problem is a maximization problem. From the definition of (OP) and (DOP), we see that $\bar{x}, \bar{y}$ are a solution of (OP) and (DOP), resp, if and only if

$A \subset\left\{x \in X \mid \varphi(x) \in\left(\varphi(\bar{x})+\mathrm{R}^{m} \backslash\left(-\mathrm{i} n t \mathrm{R}_{+}^{m}\right)\right) \cap\left(\varphi(\bar{y})+\mathrm{R}_{+}^{m}\right)\right\}$.

By substituting $\bar{x}$ and $\bar{y}$ in this inclusion, we obtain $\varphi_{i}(\bar{x})-\varphi_{i}(\bar{y}) \in \mathrm{bdR}_{+}^{m}$. Furthermore, $\bar{y}$ must be unique, but $\bar{x}$ not. Thus, we have a simple geometric explanation in the objective space $\mathrm{R}^{m}$ for (OP) and (DOP).

Now we are interested in approximations of these two dual-to-each-other problems. Corresponding to the notion of $(\varepsilon, \xi)$-approximate solutions for (EP) and (DEP), we define $(\varepsilon, \xi)$ approximate solutions for (OP) and (DOP) as follows. With $e:=(1, \ldots, 1) \in \mathrm{R}^{m}$, if

$$
\varphi\left(\bar{x}_{\varepsilon, \xi}\right)-\varphi(y)-\varepsilon \xi\left(\bar{x}_{\varepsilon, \xi}-y\right) e \notin i n t R_{+}^{m} \text {, for all } y \in A
$$


$\left(\varphi(x)-\varphi\left(\bar{y}_{\varepsilon, \xi}\right)+\varepsilon \xi\left(x-\bar{y}_{\varepsilon, \xi}\right) e \in \mathrm{R}_{+}^{m}\right.$, for all $x \in A$, resp $)$,

then $\bar{x}_{\varepsilon, \xi}\left(\bar{y}_{\varepsilon, \xi}\right.$, resp) is called an $(\varepsilon, \xi)-$ approximate weak minimizer (an $(\varepsilon, \xi)$ approximate strong minimizer, resp) of $\varphi$ on $A$. In fact, a little more general notion called an $\varepsilon$-quasi minimizer was already defined in [24] for multiobjective optimization. Here, we formulate the above two $(\varepsilon, \xi)$-approximate solutions as a special case of the corresponding definitions for (EP) and (DEP) and pay attention also on their duality.

To state consequences of Propositions 2-5 in terms of the data of (OP), we need the following definition.

\section{Definition 6}

(i) A sequence of $m$ functions $\left\{\varphi_{1}^{k}, \ldots, \varphi_{m}^{k}\right\}_{k}$, defined on $A^{k}$, in $\mathrm{fv}-\mathrm{fcn}(X)$ is called to uniformly epi-converge to $m$ limits $\varphi_{1}, \ldots, \varphi_{m}$, resp, if Definition 1 (iii) is satisfied for all $\varphi_{i}^{k}, \varphi_{i}$ and $i$, with the sequence $x^{k}$ in (b) being common for all $1 \leq i \leq m$. The definition of uniform hypoconvergence is similar.

(ii) A sequence of $m$ functions $\left\{\varphi_{1}^{k}, \ldots, \varphi_{m}^{k}\right\}_{k}$, defined on $A^{k}$, in $\mathrm{fv}$-fen $(X)$ is said to converge uniformly graphically to $m$ limits $\varphi_{1}, \ldots, \varphi_{m}$, resp, if they all graphically converge and the condition $(\beta)$ in Proposition 1 (i) is satisfied with the sequence $x^{k}$ being common for all $1 \leq i \leq m$.

(Note that, in [21] and [23], there was already the notion defined in Definition 6(i), but for the special case where $A^{k} \rightarrow A$ and these sets are convex.) We have a relation between Definition 6 and the convergence of $\Omega^{k}$ defined by the rule (5) as follows. For the sake of completeness, we provide also a proof of part (i), which is similar to that of part (a) of Proposition 5.2 in [21], but we do not assume that $A^{k}, A$ are convex and $A^{k} \rightarrow A$.

Lemma 1 (i) If $\varphi_{1}^{k}, \ldots, \varphi_{m}^{k}$ uniformly epi-converge to $\varphi_{1}, \ldots, \varphi_{m}$, then $\Omega^{k}$ defined by the rule (5) e/hconverges to $\Omega$.

(ii) If $\varphi_{1}^{k}, \ldots, \varphi_{m}^{k}$ uniformly graphically converge to $\varphi_{1}, \ldots, \varphi_{m}$, then $\Omega^{k}$ defined by the rule (5) epiconverges to $\Omega$ and $A^{k} \rightarrow A$.

Proof (i) We check first (a) in Definition 3 (of $\mathrm{e} / \mathrm{h}$-convergence). For any $x^{k} \in A^{k} \rightarrow x$, Definition 6 (i)(a) gives, for all $i, \liminf _{k} \varphi_{i}^{k}\left(x^{k}\right) \geq \varphi_{i}(x)$ if $x \in A$. By Definition 6 (i)(b), for all $y \in A$, there exists $y^{k} \in A^{k} \rightarrow y$ such that $\limsup _{k} \varphi_{i}^{k}\left(y^{k}\right) \leq \varphi_{i}(y)$ for all $i$. Hence, for all $i$, $\liminf _{k}\left(\varphi_{i}^{k}\left(x^{k}\right)-\varphi_{i}^{k}\left(y^{k}\right)\right) \geq \varphi_{i}(x)-\varphi_{i}(y)$. On the other hand, by the definition of $\Omega^{k}$, for all $k$, there is $i_{k}$ such that $\Omega^{k}\left(x^{k}, y^{k}\right)=\varphi_{i_{k}}\left(x^{k}\right)-\varphi_{i_{k}}\left(y^{k}\right)$. Since the set of indices $i$ is finite (consisting of $m$ elements), there exists an index $i_{0}$ such that $\Omega^{k}\left(x^{k}, y^{k}\right)=\varphi_{i_{0}}\left(x^{k}\right)-\varphi_{i_{0}}\left(y^{k}\right)$ for all $k$ (in a subsequence of $\mathrm{N})$. Therefore,

$$
\begin{gathered}
\liminf _{k} \Omega^{k}\left(x^{k}, y^{k}\right)=\liminf _{k}\left(\varphi_{i_{0}}^{k}\left(x^{k}\right)-\varphi_{i_{0}}^{k}\left(y^{k}\right)\right) \\
\geq \varphi_{i_{0}}(x)-\varphi_{i_{0}}(y) \geq \Omega(x, y) .
\end{gathered}
$$

If $x \notin A, \varphi_{i}^{k}\left(x^{k}\right) \rightarrow+\infty$ for all $i$, and hence $\Omega^{k}\left(x^{k}, y^{k}\right) \rightarrow+\infty$. Thus, (a) of Definition 3 is checked. Now consider (b). For all $x \in A$ and all $y^{k} \in A^{k} \rightarrow y, \quad$ (i)(a) of Definition 6 yields $\liminf _{k} \varphi_{i}^{k}\left(y^{k}\right) \geq \varphi_{i}(y)$ for all $i$ if $y \in A$, and (i)(b) gives $x^{k} \in a^{k} \rightarrow x$ with $\limsup _{k} \varphi_{i}^{k}\left(x^{k}\right) \leq \varphi_{i}(x)$ for all $i$. Consequently, for all $i$,

$$
\begin{aligned}
& \quad \limsup _{k} \Omega^{k}\left(x^{k}, y^{k}\right) \leq \limsup _{k}\left(\varphi_{i}^{k}\left(x^{k}\right)-\varphi_{i}^{k}\left(y^{k}\right)\right) \\
& \leq \varphi_{i}(x)-\varphi_{i}(y) . \\
& \text { Consequently, }
\end{aligned}
$$
$\limsup _{k} \Omega^{k}\left(x^{k}, y^{k}\right) \leq \Omega(x, y)$. If $y \notin A, \quad \varphi_{i}^{k}\left(y^{k}\right) \rightarrow+\infty$ for all $i$, which implies that $\Omega^{k}\left(x^{k}, y^{k}\right) \rightarrow-\infty$. Thus, Definition 3 is verified completely. (ii) By Proposition 1 (ii), $\varphi_{1}^{k}, \ldots, \varphi_{m}^{k}$ both epi- and hypoconverge uniformly to $\varphi_{1}, \ldots, \varphi_{m}$, and $A^{k} \rightarrow A$. We check first condition (a) for the epi-convergence of $\Omega^{k}$. For all $\left(x^{k}, y^{k}\right) \in A^{k} \times A^{k} \rightarrow(x, y)$ and indices $i$, we have both $\liminf _{k} \varphi_{i}^{k}\left(x^{k}\right) \geq \varphi(x)$ and $\limsup _{k} \varphi_{i}^{k}\left(y^{k}\right) \leq \varphi(y)$. Since the number $m$ of indices is finite, there is an index $i_{0}$ such that $\Omega^{k}\left(x^{k}, y^{k}\right)=\varphi_{i_{0}}^{k}\left(x^{k}\right)-\varphi_{i_{0}}^{k}\left(y^{k}\right)$ for all $k$ up to subsequences of $\mathrm{N}$. Hence,

$$
\begin{gathered}
\liminf _{k} \Omega^{k}\left(x^{k}, y^{k}\right)=\liminf _{k}\left(\varphi_{i_{0}}^{k}\left(x^{k}\right)-\varphi_{i_{0}}^{k}\left(y^{k}\right)\right) \\
\geq \varphi_{i_{0}}(x)-\varphi_{i_{0}}(y) \geq \Omega(x, y) .
\end{gathered}
$$

Consider now condition (b). By the uniform convergence (given by (ii)), for all $(x, y) \in A \times A$, there exists $\left(x^{k}, y^{k}\right) \rightarrow(x, y)$ such that, for all indices $i$

$$
\limsup _{k} \Omega^{k}\left(x^{k}, y^{k}\right) \leq \limsup _{k}\left(\varphi_{i}^{k}\left(x^{k}\right)-\varphi_{i}^{k}\left(y^{k}\right)\right)
$$




$$
\leq \varphi_{i}(x)-\varphi_{i}(y) \quad \text { and then }
$$

$\limsup _{k} \Omega^{k}\left(x^{k}, y^{k}\right) \leq \Omega(x, y)$.

Denote the set of the weak minimizers and $(\varepsilon, \xi)$ approximate weak minimizers of $\varphi$ on $C$ by $\mathrm{WE}(\varphi, C)$ and $\mathrm{WE}_{\varepsilon, \xi}(\varphi, A)$. We have clearly the following consequence of Proposition 3 for stability of (OP).

Proposition 7 If $\left.\varepsilon^{k}\right] \varepsilon$ and $\varphi_{1}^{k}, \ldots, \varphi_{m}^{k}$ uniformly epi-converge to $\varphi_{1}, \ldots, \varphi_{m}$, then

$$
A \cap \operatorname{Limsup}_{k} \mathrm{WE}_{\varepsilon^{k}, \xi}^{k}\left(\varphi^{k}, A^{k}\right) \subset \mathrm{W} E_{\varepsilon, \xi}(\varphi, A),
$$

i.e., any cluster point in $A$ of a sequence of $\left(\varepsilon^{k}, \xi\right)$-approximate solutions of problems ( $\left.\mathrm{OP}^{k}\right)$ is an $(\varepsilon, \xi)$-approximate solution of problem (OP).

\section{REFERENCES}

[1] D.W. Walkup and R. J-B. Wets, Continuity of some convex-cone-valued mappings. Proc. Amer. Math. Soc. 18, 229-235, 1967.

[2] R.A. Wijman, Convergence of sequences of convex sets, cones and functions. Bull. Amer. Math. Soc. 70, 186-188, 1964.

[3] R.A. Wijman, Convergence of sequences of convex sets, cones and functions II. Trans. Amer. Math. Soc. 123, 3245, 1966.

[4] H. Attouch and R. J-B. Wets, Approximation and convergence in nonlinear optimization. In Nonlinear Programming, O. Mangasarian, R. Meyer and S. Robinson, eds., Academic Press, New York, 367-394, 1981.

[5] H. Attouch and R. J-B. Wets, Convergence des points min/sup et de points fixes. Comp. Ren. Acad. Sci. Paris 296, 657-660, 1983.

[6] H. Attouch and R. J-B. Wets, A convergence for bivariate functions aimed at the convergence of saddle values. In Mathematical Theory Optimization, J. Cecconi and T. Zolezzi, eds., Springer, 1-42, 1983.

[7] A. Jofré and R. J-B. Wets, Variational convergence of bivariate functions: Lopsided convergence. Math. Program. 116-B, 275-295, 2009.

[8] A. Jofré and R. J-B. Wets, Variational convergence of bifunctions: Motivating applications. SIAM J. Optim. 24, 1952-1979, 2014.

[9] H.T.H. Diem and P.Q. Khanh, Criteria for epi/hypo convergence of finite-valued bifunctions. Vietnam J. Math. 43, 439-458, 2015.

[10] H.T.H. Diem and P.Q. Khanh, Approximations of optimization-related problems in terms of variational convergence. Vietnam J. Math. 44, 399-417, 2016.

[11] H. Attouch, Variational Convergence for Functions and Operators. Applicable Mathematics Series, Pitman, 1984

[12] R.T. Rockafellar and R.J-B. Wets, Variational Analysis. Springer, Berlin, 3rd printing edition, 2009.

[13] J. Dupa EMBED Equation.3 ov EMBED Equation.3 and R.J-B. Wets, Asymptotic behavior of statistical estimators and of optimal solutions of stochastic optimization problems. Ann. Statist. 16, 1517-1549, 1988.

[14] M.B. Lignola and J. Morgan, Generalized variational inequalities with pseudomonotone operators under perturbations. J. Optim. Theory Appl. 101, 213-220, 1999.

[15] G. G EMBED Equation.3 rkan and J.P. Pang, Approximations of Nash equilibria. Math. Program. B 117, 223-253, 2009.

[16] R. Lopez, Approximations of equilibrium problems. SIAM J. Control Optim. 50, 1038-1070, 2012.

[17] H. Attouch and R.J-B. Wets, A convergence for bivariate functions aimed at the convergence of saddle values. In Mathematical Theory Optimization, J. Cecconi and T. Zolezzi, eds., Vol. 979 of Lecture Notes in Mathematics, Springer, pp. 1-42, 1983.

[18] A. Bagh, Epi/hypo-convergence: the slice topology and saddle points approximation. J. App. Anal. 4, 13-39, 1996.

[19] S.E. Wright, Consistency of primal-dual approximations for convex optimal control problems. SIAM J. Control Optim. 33, 1489-1509, 1995.

[20] A. Iusem and W. Sosa, New existence results for equilibrium problems. Nonlinear Anal. 52, 621-635, 2003.

[21] R. Lopez, Approximations of equilibrium problems. SIAM J. Control Optim. 50, 1038-1070, 2012.

[22] I.V. Konnov and S. Schaible, Duality for equilibrium problems under generalized monotonicity. J. Optim. Theory Appl. 104, 395-408, 2000.

[23] R. Lopez and C. Vera, On the set of weakly efficient minimizers for convex multiobjective programming. Oper. Res. Lett. 36, 651-655, 2008. 


\title{
Xấp xỉ các bài toán biến phân theo kiểu hội tụ biến phân
}

\author{
Huỳnh Thị Hồng Diễm
}

Tóm tắt - Trước hết chúng tôi trình bày về hội tụ biến phân của hàm một thành phần và tính chất co bản của hội tụ này. Trong phần tiếp theo, chúng tôi mở rộng hội tụ biến phân cho hàm có miền là tích của hai tập, ta gọi là song hàm (hàm hai thành phần). Chúng tôi đưa ra định nghĩa hội tụ biến phân của song hàm xác định trên không gian metric gồm hội tụ epi/hypo, hội tụ minsup-lop và hội tụ maxinflop. Tính chất biến phân của hội tụ này cũng được xét đến ở đây. Chuyển qua nội dung chính, chúng tôi xét xấp xỉ của một số bài toán liên qua đến tối ưu hóa quan trọng và điển hình: bài toán cân bằng và bài toán tối ưu nhiều mục tiêu. Khi áp dụng vào trường họ̣p đặc biệt là các bài toán tương ứng trong không gian hữu hạn chiều, một số kết quả của chúng tôi cũng cải tiến và làm sâu thêm các kết quả tương úng đã có trước.

Từ khóa - Hội tụ biến phân, tính chất biến phân, điểm yên ngựa, bài toán cân bằng, tối ưu đa mục tiêu 